\title{
The Strict Terminal Connection Problem on Chordal Bipartite Graphs
}

\author{
Alexsander Andrade de Melo \\ Celina Miraglia Herrera de Figueiredo \\ Uéverton dos Santos Souza
}

\begin{abstract}
A strict connection tree $T$ of a graph $G$ for a non-empty subset $W \subseteq V(G)$, called terminal set, is a tree subgraph of $G$ whose leaf set coincides with $W$. A non-terminal vertex $v \in V(T) \backslash W$ is called linker if its degree in $T$ is exactly 2 , and it is called router if its degree in $T$ is at least 3 . Given a graph $G$, a terminal set $W \subseteq V(G)$ and two non-negative integers $\ell$ and $r$, the STRICT TERMINAL CONNECTION PROBLEM (S-TCP) asks whether $G$ admits a strict connection tree for $W$ with at most $\ell$ linkers and at most $r$ routers. In the present extended abstract, we prove that S-TCP is NP-complete on chordal bipartite graphs even if $\ell$ is bounded by a constant.
\end{abstract}

\section{Introduction}

STEINER TREE is one of the most fundamental problems in graph theory

2000 AMS Subject Classification: 68R10, 68Q17.

Keywords and Phrases: Connection tree, Terminal vertices, Steiner tree, Chordal bipartite graphs, NP-completeness.

This research was supported by CAPES (Finance code 001), CNPq (140399/20178, 407635/2018-1, 303726/2017-2), and FAPERJ (CNE E-26/202.793/2017, JCNE E$26 / 203.272 / 2017)$ 
and combinatorial optimization, being related to many real-world applications. In this work, we study the complexity of the so-called STRICT TERMINAL CONNECTION problem, which is a natural variation of STEINER TREE introduced by Dourado et al. [1] motivated by questions in information security, network routing and telecommunication.

Let $G$ be a graph and $W \subseteq V(G)$ be a non-empty set, called terminal set. A strict connection tree of $G$ for $W$ is a tree subgraph of $G$ whose leaf set is equal to $W$. A non-terminal vertex of a strict connection tree $T$ is called linker if its degree in $T$ is exactly 2 , and it is called router if its degree in $T$ is at least 3 . We remark that the vertex set of every strict connection tree can be partitioned into terminal vertices, linkers and routers. For each strict connection tree $T$, we let $\mathrm{L}(T)$ denote the linker set of $T$ and $\mathrm{R}(T)$ denote the router set of $T$. Next, we formally define the Strict terminal CONNECTION problem.

Strict Terminal Connection (S-TCP)

Input: $\quad$ A graph $G$, a non-empty terminal set $W \subseteq V(G)$ and two non-negative integers $\ell$ and $r$.

Question: Does there exist a strict connection tree $T$ of $G$ for $W$, such that $|\mathrm{L}(T)| \leq \ell$ and $|\mathrm{R}(T)| \leq r$ ?

Table 1 summarises the known complexity results of S-TCP with respect to the parameters $\ell, r, \Delta(G)$, and the classes of split graphs and cographs. In addition to these results, in 4], S-TCP was studied from the perspective of disjoint paths and integral commodity flow problems.

\begin{tabular}{|c|c|c|c|c|c|}
\hline \multirow[b]{2}{*}{ Graph class } & \multicolumn{5}{|c|}{ Parameters } \\
\hline & - & $\ell$ & $r$ & $\ell, r$ & $\ell, r, \Delta(\mathbf{G})$ \\
\hline General & NPC 1 & NPC 1] & $\begin{array}{c}\text { P for } r \in\{0,1\}[2] \\
\text { but W[2]h } 3]\end{array}$ & $\begin{array}{c}\text { XP 1] } \\
\text { but W[2]h [3] }\end{array}$ & $\begin{array}{c}\text { FPT 1, 3] but } \\
\text { No-poly kernel } 3\end{array}$ \\
\hline$\Delta=4$ & NPC 3 & NPC 3 & $\mathrm{P}$ for $r \in\{0,1\}$ & FPT 1, 3] & FPT 1, 3] \\
\hline$\Delta=3$ & NPC 3 & $\mathrm{XP} 3$ & $\mathrm{P}$ for $r \in\{0,1\}$ & FPT 1, 3 & FPT 1, 3] \\
\hline Split & NPC 3 & NPC 3 & $\begin{array}{c}\text { XP [3] } \\
\text { but W[2]h [3] }\end{array}$ & $\begin{array}{c}\text { XP [1] 3] } \\
\text { but W[2]h [3] }\end{array}$ & FPT 1. 3] \\
\hline Cographs & $\mathrm{P} 3]$ & $\mathrm{P}$ 3] & $\mathrm{P} 3$ & $\mathrm{P}$ 3] & P 3] \\
\hline
\end{tabular}

Table 1: Computational complexity of S-TCP. (Adapted from [3].) 
Contribution. In this work, we prove that S-TCP remains NP-complete when restricted to chordal bipartite graphs, even if $\ell \geq 0$ is bounded by a constant.

\section{S-TCP on Chordal Bipartite Graphs}

A graph $G$ is called chordal bipartite if every induced cycle of $G$ has length 4. Equivalently, a graph $G$ is chordal bipartite if $G$ is bipartite and every cycle of $G$ of length at least 6 has a chord, i.e. an edge between two non-consecutive vertices of the cycle.

To prove that S-TCP is NP-complete on chordal bipartite graphs, we present a polynomial-time reduction from VERTEx COVER, which has as input a graph $G$ and a positive integer $k$ and asks whether there is a subset $S \subseteq V(G)$ such that $|S| \leq k$ and every edge of $G$ has an endpoint in $S$. The proposed reduction, described next, is based on the polynomial-time reduction given by Müller and Brandstädt [5] so as to prove that STEINER TREE is NP-complete on chordal bipartite graphs.

Construction. Let $I=(G, k)$ be an instance of VERTEX COVER and $c \geq 0$ be a constant. Assume that $V(G)=\left\{v_{1}, \ldots, v_{n}\right\}$ for some positive integer $n \geq 2$. Moreover, assume that $G$ has at least one edge, i.e. $m=$ $|E(G)| \geq 1$. We let $f(I, c)=(H, W, \ell=c, r)$ be the instance of S-TCP defined as follows.

1. For each $v_{i} \in V(G)$, create the gadget $H_{i}$ as illustrated in Figure 1 .

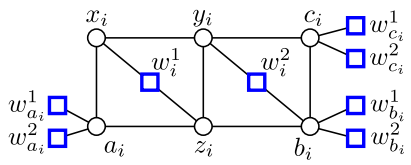

Figure 1: Gadget $H_{i}$. 
2. Subdivide the edge $w_{a_{1}}^{1} a_{1}$ of $H_{1}$ into $\ell$ new vertices $u_{1}, u_{2}, \ldots, u_{\ell}$, creating the induced path $\left\langle w_{a_{1}}^{1}, u_{1}, \ldots, u_{\ell}, a_{1}\right\rangle$.

3. For each pair $v_{i}, v_{j} \in V(G)$, with $i \neq j$, add the edges $x_{i} y_{j}$ and $z_{i} y_{j}$, making the subgraph of $H$ induced by $X \cup Y \cup Z$ a complete bipartite graph with bipartition $(X \cup Z, Y)$, where $X=\left\{x_{i} \mid v_{i} \in V(G)\right\}$, $Y=\left\{y_{i} \mid v_{i} \in V(G)\right\}$ and $Z=\left\{z_{i} \mid v_{i} \in V(G)\right\}$.

4. For each $v_{i} v_{j} \in E(G)$, create the gadgets $H_{i j}$ and $H_{j i}$ as illustrated in Figure 2,

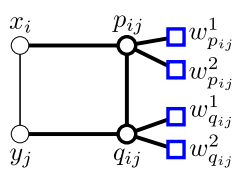

(a) $H_{i j}$

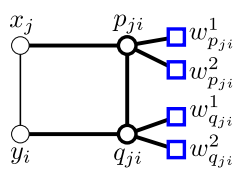

(b) $H_{j i}$

Figure 2: Gadgets $H_{i j}$ and $H_{j i}$, respectively.

5. Finally, define $W=W_{1} \cup W_{2} \cup W_{3}$ and $r=k+4 n+4 m$, where $W_{1}=\left\{w_{i}^{1}, w_{i}^{2} \mid v_{i} \in V(G)\right\}, W_{2}=\left\{w_{a_{i}}^{1}, w_{a_{i}}^{2}, w_{b_{i}}^{1}, w_{b_{i}}^{2}, w_{c_{i}}^{1}, w_{c_{i}}^{2} \mid v_{i} \in\right.$ $V(G)\}$, and $W_{3}=\left\{w_{p_{i j}}^{1}, w_{p_{i j}}^{2}, w_{q_{i j}}^{1}, w_{q_{i j}}^{2} \mid v_{i} v_{j} \in E(G)\right\}$.

Lemma 2.1. Let $I=(G, k)$ be an instance of VERTEX COVER, such that $G$ has at least one edge. For every $c \geq 0$, the graph $H$ of $f(I, c)$ is chordal bipartite.

Proof. First, we note that $H$ is chordal bipartite if and only if the graph $G^{\prime}=H-\left(W_{2} \cup W_{3}\right)$ is chordal bipartite. Indeed, the vertices belonging to $W_{2} \cup W_{3}$ are vertices of degree 1 of $H$, and therefore they do not belong to any cycle of $H$. Consequently, in order to prove this lemma, it is sufficient to show that $G^{\prime}$ is chordal bipartite. Note that, for every $v_{i} \in V(G), w_{i}^{1}$ is a false twin of $a_{i}$ in $G^{\prime}$, i.e. $N_{G^{\prime}}\left(w_{i}^{1}\right)=N_{G^{\prime}}\left(a_{i}\right)$. Similarly, for every $v_{i} \in$ $V(G), w_{i}^{2}$ is a false twin of $c_{i}$ in $G^{\prime}$, i.e. $N_{G^{\prime}}\left(w_{i}^{2}\right)=N_{G^{\prime}}\left(c_{i}\right)$. As a result, if $w_{i}^{1}$ or $w_{i}^{2}$ belongs to an odd cycle or to an induced cycle of length greater than or equal to 6 in $G^{\prime}$, then certainly $a_{i}$ or $c_{i}$, respectively, also belongs 


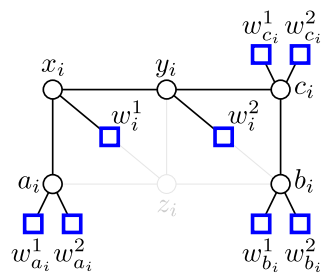

(a) $H_{i}-z_{i}$

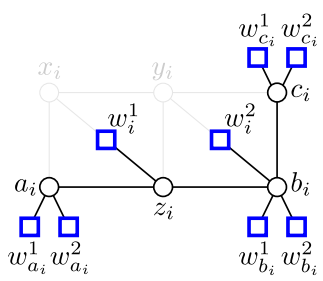

(b) $H_{i}-\left\{x_{i}, y_{i}\right\}$

Figure 3: Subgraphs $H_{i}-z_{i}$ and $H_{i}-\left\{x_{i}, y_{i}\right\}$, respectively.

to an odd cycle or to an induced cycle of length greater than or equal to 6 in $G^{\prime}$. Therefore, it follows from the fact that $H-\left(W_{1} \cup W_{2} \cup W_{3}\right)$ is chordal bipartite [5] that $G^{\prime}$ (and, thus, $H$ ) is chordal bipartite as well.

Lemma 2.2. Let $I=(G, k)$ be an instance of VERTEX COVER, such that $G$ has at least one edge. For every $c \geq 0, I$ is a yes-instance of VERTEX COVER if and only if $f(I, c)$ is a yes-instance of $S$-TCP.

Proof. First, suppose that $I$ is a yes-instance of Vertex COVER, and let $S \subseteq V(G)$ be a vertex cover of $G$ such that $|S| \leq k$. Based on $S$, we construct a strict connection tree $T$ of $H$ for $W$ as described below.

1. For each $v_{i} \in V(G)$, if $v_{i} \in S$, then add the subgraph $H_{i}-z_{i}$ (see Figure $3 \mathrm{a}$ to $T$; on the other hand, if $v_{i} \notin S$, then add the subgraph $H_{i}-\left\{x_{i}, y_{i}\right\}$ (see Figure $3 \mathrm{~b}$ ) to $T$.

2. For each $v_{i} v_{j} \in E(G)$ with $v_{i} \in S$, if $v_{j} \notin S$ or $i<j$, then add the subgraphs $H_{i j}-y_{j}$ (see Figure 4a) and $H_{j i}-x_{j}$ (see Figure 4b) to $T$. We remark that, possibly, the pairs of vertices $x_{i}$ and $y_{j}$, and $y_{i}$ and $x_{j}$, simultaneously belong to $V(T)$. However, if $x_{i} p_{i j} \in E(T)$ or $y_{i} q_{j i} \in E(T)$, then $x_{i} y_{j}, y_{j} q_{i j} \notin E(T)$ and $x_{j} y_{i}, x_{j} p_{j i} \notin E(T)$.

One can verify that, until the last step, $T$ is an acyclic subgraph of $H$ that contains all the terminal vertices belonging to $W$. Thus, in order to conclude the construction of $T$, we only need to connect the connected components of $T$ in such a way that the resulting graph is still an acyclic 


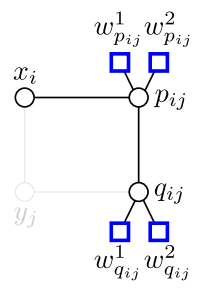

(a) $H_{i j}-y_{j}$

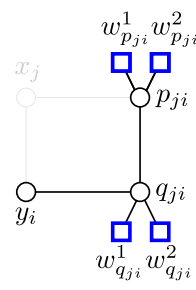

(b) $H_{j i}-x_{j}$

Figure 4: Subgraphs $H_{i j}-y_{j}$ and $H_{j i}-x_{j}$, respectively.

subgraph of $H$. Since $|E(G)| \geq 1,|S| \geq 1$. As a result, $Y_{S}=\left\{y_{i} \mid v_{i} \in S\right\}$ is non-empty. Then, let $y_{\alpha}$ be a vertex in $Y_{S}$, arbitrarily chosen. It follows from the construction of $G$ that $y_{\alpha}$ is adjacent in $G$ to all vertices belonging to $X_{S} \cup Z_{S}$, where $X_{S}=\left\{x_{i} \mid v_{i} \in S\right\}$ and $Z_{S}=\left\{z_{i} \mid v_{i} \notin S\right\}$. Moreover, note that all connected components of $T$ necessarily have at least one vertex in $X_{S} \cup Z_{S}$. Thus, to conclude the construction of $T$, we perform the following operation:

3. For each connected component $T^{\prime}$ of $T$ which does not contain the vertex $y_{\alpha}$, select arbitrarily a vertex $v \in\left(X_{S} \cup Z_{S}\right) \cap V\left(T^{\prime}\right)$ and, then, add the edge $v y_{\alpha}$ to $T$.

Then, we have finally obtained a subgraph $T$ of $H$ which is a tree and contains all the terminal vertices belonging to $W$. Moreover, note that $\mathrm{L}(T)=\left\{u_{1}, u_{2}, \ldots, u_{\ell}\right\}$ and

$$
\begin{aligned}
\mathrm{R}(T) & =\left\{x_{i}, y_{i} \mid v_{i} \in S\right\} \cup\left\{z_{i} \mid v_{i} \notin S, v_{i} \in V(G)\right\} \\
& \cup\left\{a_{i}, b_{i}, c_{i} \mid v_{i} \in V(G)\right\} \cup\left\{p_{i j}, p_{j i}, q_{i j}, q_{j i} \mid v_{i} v_{j} \in E(G)\right\} .
\end{aligned}
$$

Hence, $T$ is a strict connection tree of $G$ for $W$ such that $|\mathrm{L}(T)|=\ell$ and $|\mathrm{R}(T)|=2|S|+(n-|S|)+3 n+4 m \leq k+4 n+4 m=r$. Therefore, $f(I, c)$ is a yes-instance of S-TCP.

Conversely, suppose that $G$ admits a strict connection tree $T$ for $W$ such that $|\mathrm{L}(T)| \leq \ell$ and $|\mathrm{R}(T)| \leq r=k+4 n+4 m$. Note that, by construction of $H$, the only path in $H$ between the terminal vertices $w_{a_{1}}^{1}$ 
and $w_{a_{1}}^{2}$ contains the non-terminal vertices $u_{1}, u_{2}, \ldots, u_{\ell}$. Besides that, $d_{H}\left(u_{i}\right)=2$ for every $i \in\{1, \ldots, \ell\}$. As a result, $\mathrm{L}(T)=\left\{u_{1}, u_{2}, \ldots, u_{\ell}\right\}$. This implies that all the other non-terminal vertices of $T$ must be routers. In addition, for every $v_{i} \in V(G)$, we have that $a_{i} \in \mathrm{R}(T)$, since $a_{i}$ is the only neighbour in $H$ of the terminal vertices $w_{a_{i}}^{1}$ and $w_{a_{i}}^{2}$ and, thus, $a_{i}$ necessarily belongs to $V(T)$. Analogously, we have that $b_{i}, c_{i} \in \mathrm{R}(T)$.

Claim 2.1. Let $v_{i} \in V(G)$ and $T_{i}$ be the subgraph of $T$ induced by $V\left(H_{i}\right)$. If $y_{i} \in V(T)$, then we can assume that the degree of $y_{i}$ in $T_{i}$ is at least 3 .

Proof. First, we note that every path in $H$ between $y_{i}$ and $a_{i}$ contains $x_{i}$ or $z_{i}$. Consequently, $x_{i}$ or $z_{i}$ must belong to the path $P$ in $T$ between $y_{i}$ and $a_{i}$. It is not hard to verify that, if $x_{i} \in V(P)$, then we can assume that $x_{i}, w_{i}^{2}, c_{i} \in N_{T}\left(y_{i}\right)$. On the other hand, if $z_{i} \in V(P)$, then we can assume that $z_{i}, w_{i}^{2}, c_{i} \in N_{T}\left(y_{i}\right)$.

Claim 2.2. Let $v_{i} \in V(G)$. If $x_{i} \in V(T)$, then we can assume that $y_{i} \in V(T)$. Analogously, if $y_{i} \in V(T)$, then we can assume that $x_{i} \in V(T)$.

Proof. Suppose that $x_{i} \in V(T)$ but $y_{i} \notin V(T)$. The case in which $y_{i} \in$ $V(T)$ but $x_{i} \notin V(T)$ is analogous. Note that, the path in $T$ between $a_{i}$ and $c_{i}$ must contain $z_{i}$, which must be a router of $T$. Furthermore, by the previous claim, we can assume that, for every vertex $y_{j} \in N_{T}\left(z_{i}\right)$ with $j \neq i$, the degree of $y_{j}$ in $T_{j}$ is at least 3 , where $T_{j}$ denotes the subgraph of $T$ induced by $V\left(H_{j}\right)$. Thus, let $T^{\prime}$ be the graph with vertex set $V\left(T^{\prime}\right)=V(T) \backslash\left\{z_{i}\right\} \cup\left\{y_{i}\right\}$ and edge set

$$
\begin{aligned}
E\left(T^{\prime}\right) & =E(T) \backslash\left(\left\{v z_{i} \mid v \in N_{T}\left(z_{i}\right)\right\} \cup\left\{w_{i}^{2} b_{i}\right\}\right) \\
& \cup E\left(H_{i}-z_{i}\right) \cup\left\{y_{j} x_{i} \mid y_{j} \in N_{T}\left(z_{i}\right), j \neq i\right\} .
\end{aligned}
$$

One can verify that $T^{\prime}$ is a strict connection tree of $H$ for $W$ that simultaneously contains the vertices $x_{i}$ and $y_{i}$ and satisfies the constraints $\left|\mathrm{L}\left(T^{\prime}\right)\right| \leq \ell$ and $\left|\mathrm{R}\left(T^{\prime}\right)\right| \leq r$; more precisely, $\mathrm{L}\left(T^{\prime}\right)=\mathrm{L}(T)$ and $\mathrm{R}\left(T^{\prime}\right)=$ $\left(\mathrm{R}(T) \backslash\left\{z_{i}\right\}\right) \cup\left\{y_{i}\right\}$. 
Thus, consider the subset $S=\left\{v_{i} \in V(G) \mid x_{i}, y_{i} \in V(T)\right\}$. We claim that $S$ is a vertex cover of $G$. For the sake of contradiction, suppose that there exists an edge $e=v_{i} v_{j} \in E(G)$ such that $S \cap\left\{v_{i}, v_{j}\right\}=\emptyset$. Consequently, $x_{i}, y_{i} \notin V(T)$ and $x_{j}, y_{j} \notin V(T)$. Moreover, we have that $w_{p_{i j}}^{1}, w_{p_{i j}}^{2}, w_{q_{i j}}^{1}, w_{q_{i j}}^{2} \notin V(T)$ (as well as $w_{p_{j i}}^{1}, w_{p_{j i}}^{2}, w_{q_{j i}}^{1}, w_{q_{j i}}^{2} \notin V(T)$ ), since $T$ is connected and the only path in $T$ between such terminals and any other terminal belonging to $W$ - for example, the terminals in $W_{1} \cup W_{2}$ - necessarily contains $x_{i}$ or $y_{j}\left(x_{j}\right.$ or $y_{i}$, respectively). However, this contradicts the hypothesis that $W_{3} \subseteq W \subseteq V(T)$. As a result, such an edge $e$ cannot exist. In other words, $S$ is a vertex cover of $G$. Finally, note that, if $|S|=k^{\prime}$, then

$$
|\mathrm{R}(T)|=2 k^{\prime}+\left(n-k^{\prime}\right)+3 n+4 m=k^{\prime}+4 n+4 m \leq r=k+4 n+4 m,
$$

which implies $|S| \leq k$. Therefore, $I$ is a yes-instance of VERTEx COVER.

Theorem 2.1. $S$-TCP remains NP-complete when restricted to chordal bipartite graphs, even if $\ell$ is bounded by a constant.

Proof. This result follows from Lemmas 2.1 and 2.2 and from the fact that the construction $f$ can be computed in polynomial-time over the input size of the given instance $I$ of VERTEX COVER and the parameter $\ell$.

\section{Concluding Remarks}

In the present extended abstract, we have proved that S-TCP is NPcomplete on chordal bipartite graphs even if $\ell$ is bounded by a constant. On the other hand, it remains unknown whether S-TCP can be solved in polynomial-time on chordal bipartite graphs if $r$ is bounded by a constant (and $\ell$ is arbitrarily large). More generally, one of the main questions concerning S-TCP is whether the problem parameterized by $r$ is in XP. 


\section{References}

[1] M. C. Dourado, R. A. Oliveira, F. Protti, and U. S. Souza, Conexão de terminais com número restrito de roteadores e elos, In Proceedings of XLVI Simpósio Brasileiro de Pesquisa Operacional (2014), pp. 29652976.

[2] A. A. Melo, C. M. H. de Figueiredo, and U. S. Souza, Connecting terminals using at most one router, Matemática Contemporânea $\mathbf{4 5}$ (2017), SBM, pp. 49-57.

[3] A. A. Melo, C. M. H. de Figueiredo, and U. S. Souza, A multivariate analysis of the strict terminal connection problem, Journal of Computer and System Sciences 111 (2020), pp. 22-41.

[4] A. A. Melo, C. M. H. de Figueiredo, and U. S. Souza, On undirected two-commodity integral flow, disjoint paths and strict terminal connection problems, Networks 77 (2021), pp. 559-571.

[5] H. Müller and A. Brandstädt, The NP-completeness of Steiner tree and dominating set for chordal bipartite graphs, Theoretical Computer Science 53 (1987), pp. 257-265.

Alexsander Andrade de Melo Federal University of Rio de Janeiro

Rio de Janeiro, Brazil.

aamelo@cos.ufrj.br

Uéverton dos Santos Souza

Fluminense Federal University

Niterói, Brazil.

ueverton@ic.uff.br
Celina Miraglia Herrera de

Figueiredo Federal University of Rio de Janeiro Rio de Janeiro, Brazil. celina@cos.ufrj.br 